\title{
Research on the Influence of Multi-ball Training in Tennis Forehand Stroke Learning
}

\author{
Zhou Zheng \\ Oxbridge College, Kunming University of Science and Technology, Kunming, Yunnan 650106
}

\begin{abstract}
Keywords: Multi ball training; Tennis; Forehand stroke technique; Learning effects
\end{abstract}
\begin{abstract}
As one of the fashionable sports, tennis is popular with people, and people pay more attention to the developments of sports, tennis has gained great growth from quantity to quality. Tennis, as a sports item, it uses scientific method and technology in training while developing itself, which becomes the main mode of tennis learning. With growing of society, tennis batting technique also changes as time goes by, as for beginners, forehand stroke is a basic batting way to cross the threshold, and for many tennis trainers, it also an important measure to win score. As a basic skill, forehand stroke gained highly attention among tennis trainers. Since tennis is going to complete gradually, forehand stroke employs multi-ball training, which now is a very common learning mode. Therefore, this article mainly explores the influence of multi-ball training in tennis forehand stroke. Among ball sports items, multi-ball training is very common, it could not only promote the physical quality players, but also a usual method to face future strategy. Multi-ball training could trace back the 60s of last century, and since the time went by, it has been employed mostly in ball sports items with wide practical application value. As a competitive sport item, to master the norm of action is vital for tennis, using sense training to acquire different speed, power and angle which could improve the effect. And players could take short-term quick training to increase the hitting times, doing dense training and adjust training strength, normalize the hitting action that assure the solid degree of basic skills. These for tennis trainers have strong practical application value.
\end{abstract}

\section{Forehand Stroke Technique}

As for tennis learners, forehand stroke is base for tennis learning, first is to throw the ball in place and then do the hitting practice at the fixed point of net, doing hitting practice at the moving point of net as next step, last is to do irregular hitting practice. According to the practical demand of tennis trainers, at the different training stage, they could adjust corresponding stuff like sequential scans, training route or exercise spots, which could promote training effect.

\section{Principle of Multi-ball Training}

Tennis learners will have problem in using forehand stroke and understanding the concept of tennis training at the very beginning, some tennis learners is worse in basic skills that leads to a wrong learning zone, such as nonstandard hitting action and hard to grasp the key points of hitting. As to such learning situation, learners will lack efficiency in catching the ball if their coach employs pair training, it will waste time in picking balls and then leads to worse study effect. It is not good to waste time on practical exercise while concerning future of learners. However, multi-ball training will change this issue with effect. With this method, learners could reach the ideal training situation just in limit time, they will hit faster and more, enforcing the basic skills and thus promote hitting technique.

From Quantity to Quality. It is a long term to improve tennis skill, which needs day-by-day training and then promote skills fundamentally. Multi-ball training mainly use fixed point form to exercise, with it, tennis learners could improve proficiency of hitting by repeated practice, and then normalize their action, they will reach the stage from quantity to quality by long-term training ${ }^{[1]}$.

From the Elementary to the Profound. Tennis training acquires the principle that from elementary to the profound, it emphasizes tennis could not reach the high level in short time, it should combine the practical situation and thus add to corresponding training contend. In different 
learning stage, multi-ball training is also different in practical application. As for beginners, they need to control the potency dimension, and transform slow to quick. While the skills improved a bit, learners could increase the speed of follow-through and the difficulty level of actions. Tennis training should follow the principle that from surface to the deep and control the degree of easy and hard mode. $s$

\section{Function of multi-ball training}

As to tennis learning, it needs long-term training then could reach the aim at flexible using. At pre-stage, the main task of tennis learning is a rough master of this sports, and could correct the wrong actions, and then to enforce and compete these actions. Only grasp actions in every stage, will learners study smoothly.

At the Stage of Rough Master. Forehand stroke at stage of rough master uses multi-ball training, as the passers, they need to pass throw the ball to a fixed zone in continuous, tennis learners take forehand stroke training at a fixed point, at the same time, they should assure that their actions are normal which could achieve the aim of shaping norm, building the concept of muscle ${ }^{[2]}$. The passers will make sure the ball they are passing is at average potency and they will also slow the speed, controlling the frequency. According to the real demand during learning, the passers could increase training times, and adjust passing speed, potency and frequency. If potency enforced, the following speed should also be faster, thus learners will quicken frequency of hitting. In the practical training, in order to assure the training time, learners should be divided in 5 groups, and each groups should take ball training over 20, thus the basic technique of learners will be assured, to reach the ideal situation.

At the Stage of Normalizing the Action. While doing training of normalizing the actions, since tennis learners have finished the cross-threshold task that they mastered some basic technique, and different with last stage of training, in this stage, tennis training will not only keep in fixed zone, thus multi-ball training also need to take corresponding adjustment ${ }^{[3]}$. As to passers, while they in the multi-ball training, they could switch the spots of falling or change the route of throwing, to do corresponding adjustment could foster their ability of reacting in every unforeseen moment. At this stage, tennis coach should pay more attentions to enforce learners' smooth in actions that leads to a fluent hitting, during hitting, to make sure learners could control the hitting power and then bat the ball into fixed zone. Tennis coach should arouse positivity of learners, developing every action of tennis training, and after each stroke, they could make sure learners are ready to next one, which will improve the frequency of stroke ${ }^{[4]}$.

At the Stage of Enforcing and Completing the Hitting Action. At this stage, we could treat this as improving stage of tennis training, according to individual difference to increase the difficulty of multi-ball training that combined with reality, we should not improve too much in short time, however, it will not only bring nonsense to tennis learners, but also leads to a opposite effect that learners have no progression. The passers could increase the difficulty of passing and they could also combine forms of bottom and mid-field, making rule to the route of return path, such as to combine forms of straight and slant line ${ }^{[5]}$. Besides we could apply many way to operate multi-ball training, for example, to take form of targeted alignment that need to set markers on floor and then learners should hit precisely the markers, with repeated exercise, tennis learners will promote their hitting levels and at during that, coaches also need to pay attention the actions and potency of learners, and thus ask the correct to assure normalization of hitting.

\section{The application of multi-ball training in forehand stroke learning}

Multi-ball training is highly applied among tennis training, since this method is convenient and quick, and could improve technique level of tennis in short term, it has popular with many tennis learners. As a common technique in tennis learning, to take multi-ball method in practical training could have vivid effect to forehand stroke. There are two main contends taken by forehand stroke as below: 
To Throw From Bottom Line. The technique of throwing from bottom line needs hitting player to stand by behind bottom line, and ahead of them on the right is the toss, the latter should do training of throw balls in fixed place. As basic exercise form of multi-ball training, using method of throwing from bottom line could control the ball speed nicely, and make sure the actual falling spots, at this stage, learners no need to move and they should be assured to adjust such training mode ${ }^{[6]}$. Such multi-ball training is wide use in forehand stroke that could assure learners grasp key points of tennis technique. The traits of bottom-line throwing technique as follow, first, it could have reasonable control to ball speed and assure the speed of ball will slow to ensure the falling spots, in this stage, learners no need to move, to ensure the hitting spots will enforce the hitting action. It is needed to pay attention that in the practical training we should add difficulty in reasonable degree, from elementary to the profound, step by step. At the very beginning of training, hand should with ball and keep downward, to ensure ball could drop with average speed, and then switch the route gradually, next, with palm side up, tennis ball will drop slowly in the power of upwards-speed, such training method could improve velocity ${ }^{[7]}$. During forehand stroke training, the passers could hit the ball into learners that in the right side ahead of them, however, the passers and learners need tacit and then could improve practical effect.

To Pass Ball with Tennis Racket in. Such method is used when learners stay a plain developing situation, it could judge the falling spots and coming direction according to the practical passing situation, and learners could make whole hitting process smooth if they grasp frequency of hitting, therefore, this method applied widely. Such method has its own characteristics, such as passing ball with quicker speed, falling spots and direction are not fixed, balls will choose various running path in real training, all of these made it more effect ${ }^{[8]}$.

\section{Influence of applying multi-ball training in forehand stroke learning}

Multi-ball training are wild used in forehand stroke learning, and it has wide functional value, meanwhile, it also bring certain influence to forehand stroke learning. Multi-ball training has its own superiority, it could bring fine training environment to many tennis learners in limit ground, and it also improve the training frequency to ensure tennis learners could grasp forehand stroke in short time that learning efficiency will play out. Apart from these, multi-ball training is good to normalize hitting actions, and could set the right movements, meanwhile, it could also ensure stability of hitting and improve deep of hitting. In the moment of catching, learners should make precise judge, and, learners should catch the ball from the passers according to their different method of serving a ball, and then they could ensure the coherence of whole hitting action, it is important to improve the whole hitting technique ${ }^{[9]}$.

While applying multi-ball training in forehand stroke, if we only take forehand stroke training, it will bored learners, and thus they lose interest to it, meanwhile, when they passing balls with another, for tennis freshman, it is hard to hit back the ball, they have to waste time on picking ball that fell. Such situation will affect the whole efficiency of forehand stroke learning ${ }^{[10]}$. To use multi-ball training could change some issue, and bring more learning time to many tennis learners that could improve learning efficiency and has key meaning to a further study of forehand stroke.s

\section{Summary}

With the developing of our economy and comprehensive national strength, our country began paying more attentions to the development of sports filed, the age of public fitness is coming, and people will acknowledge the importance of sport. As a modern sports activity, tennis is popular by people from every generation, and forehand hitting of tennis training is the very basic hitting technique, which is meaningful to the beginners. Employing multi-ball training made study of forehand stroke easy and simple, it could also improve learning efficiency. 


\section{Reference}

[1] Duan Judong. On Improving the Technical Method of Tennis Forehand [J]. Boxing \& Fight,2016(24):59.

[2] Yue Mingxiao, LI Qingyou, Liu Yuteng. The Influence of Multi Ball Training on Tennis Batting Technology Learning [J]. Bulletin of Sport Science \& Technology

,2017(2):53-55.

[3] Xi Wenting. Analysis on the Technical Difficulties of Forehand Batting of Tennis Beginners [J]. Boxing \& Fight,2018(2):53.

[4] Han Cuicui. Study on the Teaching of Tennis Forehand Stroke [J]. SPORTS TIME,2017(4):131.

[5] Meng Yunguang, Wang Li, Wang Yong. Experimental Research on Forehand Stroke Teaching in Tennis Courses in Colleges and Universities [J]. Science \& Technology of Stationery \& Sporting Goods,2017(13):158-159.

[6] Yang Bin, Wang Qian. Biomechanical Study of Touching Ball in Tennis Forehand Stroke [J]. Journal of Inner Mongolia Normal University(Natural Science Edition),2018(1):86-89.

[7] Zhang Lianzhou. Experimental Study on the Influence of Different Intensity of Multi Ball Training on Tennis Forehand Hitting Technique $[\mathrm{J}]$. Journal of Pingxiang College,2017(4):109-111.

[8]Cao Fan. The Present Situation and Analysis of Tennis Forehand Stroke Skills [J]. Contemporary Sports Technology,2016(23):40-40,42.

[9] Ye Xin. Experimental Study of Cooperative Teaching Method in Improving Tennis Forehand Stroke Teaching Effect[J]. Journal of Zhaoqing University,2017(2):97-100.

[10] Yin Shanshan. Teaching Analysis of Forehand Stroke in Tennis Teaching of Physical Education Major [J].SPORTS TIMES,2017(15):188. 